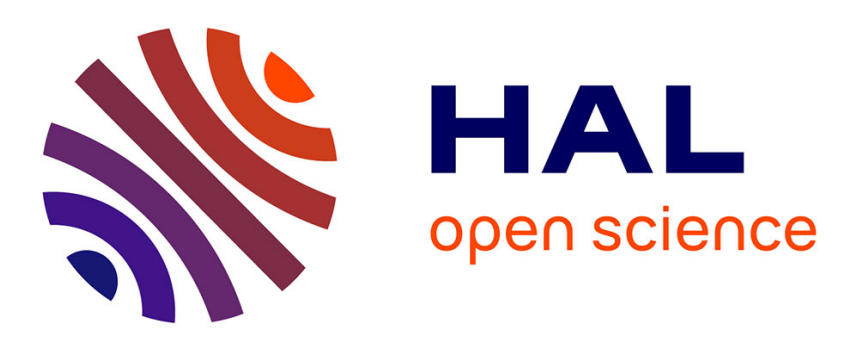

\title{
The unnormalized Dempster's rule of combination: a new justification from the Least Commitment Principle and some extensions
}

Frédéric Pichon, Thierry Denoeux

\section{- To cite this version:}

Frédéric Pichon, Thierry Denoeux. The unnormalized Dempster's rule of combination: a new justification from the Least Commitment Principle and some extensions. Journal of Automated Reasoning, 2010, 45 (1), pp.61-87. hal-00482006

\section{HAL Id: hal-00482006 https://hal.science/hal-00482006}

Submitted on 7 May 2010

HAL is a multi-disciplinary open access archive for the deposit and dissemination of scientific research documents, whether they are published or not. The documents may come from teaching and research institutions in France or abroad, or from public or private research centers.
L'archive ouverte pluridisciplinaire HAL, est destinée au dépôt et à la diffusion de documents scientifiques de niveau recherche, publiés ou non, émanant des établissements d'enseignement et de recherche français ou étrangers, des laboratoires publics ou privés. 


\title{
The Unnormalized Dempster's Rule of Combination: \\ a New Justification from the Least Commitment Principle and some Extensions*
}

\author{
Frédéric Pichon ${ }^{1}$, Thierry Denœux ${ }^{2}$ \\ 1 Thales Research and Technology, \\ Campus Polytechnique, \\ 1 avenue Augustin Fresnel, \\ 91767 Palaiseau cedex, France. \\ ${ }^{2}$ Université de Technologie de Compiègne, \\ UMR CNRS 6599 Heudiasyc, \\ Centre de Recherches de Royallieu, \\ BP20529, 60205 Compiègne Cedex, France.
}

Frederic.Pichon@thalesgroup.com

Thierry.Denoeux@utc.fr

May 7, 2010

*This paper is an extended and revised version of [25]. 


\begin{abstract}
In the Transferable Belief Model, belief functions are usually combined using the unnormalized Dempster's rule (also called the TBM conjunctive rule). This rule is used because of its intuitive appeal and because it has received formal justifications as opposed to the many other rules of combination that have been proposed in the literature. This article confirms the singularity of the TBM conjunctive rule by presenting a new formal justification based on (1) the canonical decomposition of belief functions, (2) the least commitment principle and (3) the requirement of having the vacuous belief function as neutral element of the combination. A similar result is also presented for the TBM disjunctive rule. Eventually, the existence of infinite families of rules having similar properties as those two rules is pointed out.
\end{abstract}

Keywords: Transferable Belief Model, Dempster-Shafer theory, Information fusion, Evidence theory, Belief functions, Uncertain reasoning, Combination rules. 


\section{Introduction}

The last thirty years have seen the emergence of the Dempster-Shafer theory of belief functions $[4,33,35]$, which has gained increasing interest as a conceptual framework for modeling partial knowledge and reasoning under uncertainty. Different interpretations of this theory have been proposed [38]. In particular, the Transferable Belief Model (TBM) interpretation, which will be adopted in this paper, views belief functions as representing beliefs held by rational agents. In contrast to other interpretations (based, e.g., on random sets or imprecise probabilities), the TBM does not assume any underlying probability concepts $[42,43]$. It has been successfully applied to various problems such as pattern recognition [5, 7], classifier fusion [28, 24], and military applications $[2,31,1]$. Most of these applications involve fusing belief functions and rely critically on combination rules. Although many such rules have been proposed in the literature (see $[32,41]$ for recent surveys), it appears that belief functions are usually combined in the TBM using the unnormalized version of Dempster's rule [4], referred to as the TBM conjunctive rule in this paper. An explanation to this fact is that this rule has received formal justifications for its origin and uniqueness $[9,36,18,19,16]^{1}$. It seems indeed reasonable to favor a principled rule over "ad hoc" ones [41], hence the necessity for such justifications.

A limitation, which applies to both Dempter's rule and the TBM conjunctive rule, is the requirement that the items of evidence combined be distinct, or in other words, that the information sources be independent. Some authors $[22,14,3,8]$ have attempted to address this issue. However, those proposals are either restricted to particular classes of belief functions or do not possess desirable properties such as associativity. Recently, Denœux [6] proposed a rule, called the cautious rule, for the combination of nondistinct bodies of evidence. The term cautious is reminiscent of the derivation of the rule, which is based on the least commitment principle (LCP) [37]. The LCP stipulates that one should never give more beliefs than justified by the available information, hence it promotes a cautious attitude. The cautious rule is based on the conjunctive weight function [39], which is an equivalent representation of a nondogmatic belief function arising from its canonical decomposition. The TBM conjunctive rule can also be expressed using the conjunctive weight function, which makes it interesting to study rules based on this rarely exploited function.

\footnotetext{
${ }^{1}$ One should note that, although those justifications use loosely the expression "Dempster's rule", they actually prove the unicity of the unnormalized version of Dempster's rule. Furthermore, they are not based on probability concepts, hence they can readily be used to justify the TBM conjunctive rule as already remarked by Smets [44, 41].
} 
One of the main differences between the cautious rule and the TBM conjunctive rule is that the former has no neutral element, whereas the latter admits the vacuous belief function as neutral element. This last property is quite natural for a conjunctive operator, as the vacuous belief function encodes ignorance. Hence, rules based on the conjunctive weight function and that admit the vacuous belief function as neutral element are of particular interest. The main result presented in this paper is that, among those rules, the TBM conjunctive rule is the least committed one. This can be seen as a new justification of the TBM conjunctive rule for combining nondogmatic belief functions, as this rule respects a central principle of the TBM. A counterpart to this result is also obtained for the TBM disjunctive rule [10, 37].

Despite the importance of formal justifications, one should note that some rules of combination that are not completely well founded theoretically may still be useful for some problems, such as classifier fusion (see, e.g., [29, 30]). In [9], Dubois and Prade emphasize this idea by arguing that having only one rule is "not very fortunate in the scope of AI, where one tries to simulate the human mind rather than force behavior rules issued from formal arguments" [9]. Indeed, the "descriptive nature of AI" [9] calls for more flexibility in the choice of combination rules, provided that those other rules satisfy at least a few basic and reasonable requirements. Interestingly, it is possible to cast the main result of this paper and the differences between the TBM conjunctive rule and the cautious rule in a more general context: the cautious rule can be seen as a member of an infinite family of combination rules based on t-norms $[20]$ on $(0,+\infty]$, whereas the TBM conjunctive rule belongs to an infinite family of combination rules based on uninorms [47] on $(0,+\infty]$ having one as neutral element. Furthermore, both rules have a special position in their respective family: they are the least committed elements. The relevance of the introduction of those families of rules in this paper may thus be seen as twofold. First and foremost, it allows the main result of the paper to be put in a broader perspective. Second, it also provides an answer to the need for flexibility in terms of combination rules. Demonstrating the practical usefulness of those families of rules is nonetheless beyond the scope of this paper. References [29, 30] may be cited as encouraging preliminary steps in this direction.

The rest of this paper is organized as follows. Necessary notions, such as the conjunctive and disjunctive weight functions, the LCP, and the cautious rule are first recalled in Section 2. The main result of this paper is presented in Section 3. Section 4 introduces infinite families of combination rules based on generalized t-norms and uninorms. Section 5 concludes the paper. 


\section{Fundamental Concepts}

For the reader's convenience, this paper is self-contained. Section 2.1 summarizes basic concepts and terminology related to belief functions. The conjunctive and disjunctive canonical decompositions of belief functions and the resulting conjunctive and disjunctive weight functions are then recalled in Section 2.2. This section reviews only necessary material, further recent findings on the weight functions may be found in [6]. Section 2.3 focuses on the relative informational content of belief functions. Finally, Section 2.4 summarizes the relevant parts of [6] related to the cautious rule and its dual, the bold rule.

\subsection{The TBM: Basic Definitions and Notations}

In this paper, the TBM $[42,43]$ is accepted as a model to quantify uncertainties based on belief functions [33]. In this model, the beliefs held by an agent $A g$ on a finite frame of discernment $\Omega=\left\{\omega_{1}, \ldots, \omega_{K}\right\}$ are represented by a basic belief assignment (BBA) $m$ defined as a mapping from $2^{\Omega}$ to $[0,1]$ verifying $\sum_{A \subseteq \Omega} m(A)=1$. Subsets $A$ of $\Omega$ such that $m(A)>0$ are called focal sets of $m$. The vacuous BBA, denoted $m_{\Omega}$, is defined by $m_{\Omega}(\Omega)=1$ : it corresponds to complete ignorance. A BBA is said to be dogmatic if $\Omega$ is not a focal set.

A BBA $m$ is said to be normal ${ }^{2}$ if $\emptyset$ is not a focal set, and subnormal otherwise. A subnormal BBA $m$ can be transformed into a normal BBA $m^{*}$ by the normalization operation defined as follows:

$$
m^{*}(A)= \begin{cases}k \cdot m(A) & \text { if } A \neq \emptyset \\ 0 & \text { otherwise }\end{cases}
$$

for all $A \subseteq \Omega$, with $k=(1-m(\emptyset))^{-1}$.

Equivalent representations of a BBA $m$ exist. In particular the belief, implicability, plausibility and commonality functions are defined, respectively, as:

$$
\begin{gathered}
\operatorname{bel}(A)=\sum_{\emptyset \neq B \subseteq A} m(B), \\
b(A)=\operatorname{bel}(A)+m(\emptyset), \\
p l(A)=\sum_{B \cap A \neq \emptyset} m(B),
\end{gathered}
$$

\footnotetext{
${ }^{2}$ Although the terms "normal" and "Gaussian" are used interchangeably in probability theory, the notion of normal belief function recalled here should not be confused with that of Gaussian belief function [23], which is a generalization of a Gaussian probability distribution.
} 
and

$$
q(A)=\sum_{B \supseteq A} m(B)
$$

for all $A \subseteq \Omega$. We note that functions $b$ and bel coincide when $m(\emptyset)=0$. However, these two functions need to be distinguished in the subnormal case: bel has easier interpretation in terms of degrees of belief, whereas $b$ plays a more technical role. The BBA $m$ can be recovered from any of these functions. In particular, we have:

$$
m(A)=\sum_{B \supseteq A}(-1)^{|B|-|A|} q(B),
$$

for all $A \subseteq \Omega$ and where $|A|$ denotes the cardinality of $A$.

The negation (or complement) $\bar{m}$ of a BBA $m$ is defined as the BBA verifying $\bar{m}(A)=m(\bar{A}), \forall A \subseteq \Omega$, where $\bar{A}$ denotes the complement of $A$ [10]. It can be shown that the implicability function $\bar{b}$ associated to $\bar{m}$ and the commonality function $q$ associated to $m$ are linked by the following relation:

$$
\bar{b}(A)=q(\bar{A}), \quad \forall A \subseteq \Omega .
$$

The TBM conjunctive rule is noted (). It is defined as follows. Let $m_{1}$ and $m_{2}$ be two BBAs, and let $m_{1 @ 2}$ be the result of their combination by (๑) We have:

$$
m_{1 @ 2}(A)=\sum_{B \cap C=A} m_{1}(B) m_{2}(C), \quad \forall A \subseteq \Omega .
$$

Dempster's rule, noted $\oplus$, is just the equivalent to the TBM conjunctive rule followed by normalization using (1). Both rules are commutative, associative and admit a unique neutral element: the vacuous BBA. The TBM conjunctive rule has a simple expression in terms of commonality functions. We have:

$$
q_{1} @ 2(A)=q_{1}(A) \cdot q_{2}(A), \quad \forall A \subseteq \Omega \text {. }
$$

Let us now assume that $m_{1 @ 2}$ has been obtained by combining two BBAs $m_{1}$ and $m_{2}$, and then we learn that $m_{2}$ is in fact not supported by evidence and should be "removed" from $m_{1 @ 2}$. This operation is called decombination [39] or removal [34]. It is well defined if $m_{2}$ is nondogmatic. Let $@$ denote this operator. We can write:

$$
m_{1 @ 2} @ m_{2}=m_{1}
$$

Let $q_{1}$ and $q_{2}$ be the commonality functions of two BBAs $m_{1}$ and $m_{2}$, the decombination is defined as follows:

$$
q_{1 \otimes 2}(A)=\frac{q_{1}(A)}{q_{2}(A)}, \quad \forall A \subseteq \Omega .
$$


Note that $q_{2}(A)>0$ for all $A$ as long as $m_{2}$ is nondogmatic.

A disjunctive rule (a) also exists [10, 37]. It is defined as:

$$
m_{1(1) 2}(A)=\sum_{B \cup C=A} m_{1}(B) m_{2}(C), \quad \forall A \subseteq \Omega .
$$

This rule, called the TBM disjunctive rule in this paper, has a simple expression in terms of implicability functions, which is the counterpart of (3):

$$
b_{1(0)}(A)=b_{1}(A) \cdot b_{2}(A), \quad \forall A \subseteq \Omega \text {. }
$$

The TBM disjunctive rule is commutative, associative and admits a unique neutral element: the BBA which assigns the total mass of belief to the empty set, i.e., $m(\emptyset)=1$. This BBA, which we note $m_{\emptyset}$, is the negation of the neutral BBA $m_{\Omega}$ of the TBM conjunctive rule and is sometimes called the or-vacuous BBA [40]. As for the TBM conjunctive rule, an inverse operation may be defined for the TBM disjunctive rule:

$$
b_{1(\phi)} 2(A)=\frac{b_{1}(A)}{b_{2}(A)}, \quad \forall A \subseteq \Omega .
$$

This operation is well-defined as long as $m_{2}$ is subnormal (in which case we have $b_{2}(A)>$ 0 for all $A$ ). However, it does not necessarily produce a belief function. Its interpretation is similar to that of $@$ : it removes, or "decombines", evidence which has been combined disjunctively with prior knowledge.

The dual nature of $(0$ and (a) becomes apparent when one notices that these operators are linked by De Morgan's laws [10]:

$$
\begin{aligned}
& \overline{m_{1} \circlearrowleft m_{2}}=\overline{m_{1}} \overline{m_{2}} \\
& \overline{m_{1} @ m_{2}}=\overline{m_{1} @ \overline{m_{2}}}
\end{aligned}
$$

As remarked by Smets [37], the TBM conjunctive rule is based on the assumption that the belief functions to be combined are induced by reliable sources of information, whereas the TBM disjunctive rule only assumes that at least one source of information is reliable, but we do not know which one (see also [15]). Both rules assume the sources of information to be independent (i.e., they are assumed to provide distinct, non overlapping pieces of evidence).

\subsection{Canonical Decompositions of Belief Functions}

\subsubsection{Conjunctive Weight Function}

According to Shafer [33], a BBA is said to be simple if it has the following form

$$
\begin{aligned}
& m(A)=1-w_{0} \\
& m(\Omega)=w_{0},
\end{aligned}
$$


for some $A \subset \Omega$ and some $w_{0} \in[0,1]$. Let us denote such a BBA as $A^{w_{0}}$. The vacuous BBA may thus be noted $A^{1}$ for any $A \subset \Omega$. It is clear that

$$
A^{w_{0}} @ A^{w_{0}^{\prime}}=A^{w_{0} w_{0}^{\prime}} .
$$

A BBA may be called separable if it can be obtained as the result of the combination of simple BBAs using the TBM conjunctive rule. It can then be written:

$$
m=\bigcirc_{A \subseteq \Omega} A^{w(A)},
$$

with $w(A) \in[0,1]$ for all $A \subset \Omega$.

Smets [39] showed that any nondogmatic BBA $m$ may be uniquely expressed as the decombination of two separable BBAs:

$$
m=\left(\left(_{A \subset \Omega} A^{w_{C}(A)}\right) \otimes\left(\cap_{A \subset \Omega} A^{w_{D}(A)}\right)\right.
$$

with $w_{C}(A) \in(0,1], w_{D}(A) \in(0,1]$ and $\max \left(w_{C}(A), w_{D}(A)\right)=1$ for all $A \subset \Omega$. Equation (4) is referred to as the conjunctive canonical decomposition of $m$. Let $w$ denote the mapping from $2^{\Omega} \backslash \Omega$ to $(0,+\infty)$ defined as

$$
w(A)=\frac{w_{C}(A)}{w_{D}(A)}, \quad \forall A \subset \Omega .
$$

Function $w$ is called the conjunctive weight function ${ }^{3}$ associated to $m$ [6]. If $m$ is separable, then $w_{D}(A)=1$ and $w(A) \leq 1$ for all $A \subset \Omega$. Otherwise, $w(A)>1$ for some $A \subset \Omega$. For any nondogmatic BBA $m$, we may write

$$
m=\bigcirc_{A \subseteq \Omega} A^{w(A)},
$$

where $A^{w(A)}$ is a simple BBA if $w(A) \leq 1$, and a "generalized simple BBA" (which is not a BBA) if $w(A)>1$. The conjunctive weight function is a new equivalent representation of a nondogmatic BBA, which may be computed directly from $m$ as follows:

$$
\ln w(A)=-\sum_{A \subseteq B}(-1)^{|B|-|A|} \ln q(B), \quad \forall A \subset \Omega .
$$

We notice the similarity with (2). Hence, as pointed out in [6], any procedure suitable for transforming $q$ to $m$ can be used to compute $\ln w$ from $-\ln q$.

Finally, we note that function $w$ has a simple property with respect to the TBM conjunctive rule. Let $w_{1}$ and $w_{2}$ be two weight functions, and let $w_{1 @ 2}$ denote the result of their $@$-combination. Then the following relation holds:

$$
w_{1 @ 2}(A)=w_{1}(A) w_{2}(A), \quad \forall A \subset \Omega .
$$

\footnotetext{
${ }^{3}$ We note that function $w$ is only defined for strict subsets of $\Omega$, as the notation $\Omega^{w_{0}}$ corresponds to the vacuous BBA no matter the value assigned to $w_{0}$ : consequently, $w(\Omega)$ could be fixed arbitrarily. In [17], the authors propose a particular way to extend function $w$ to $2^{\Omega}$. However, this extension will not be needed in this paper.
} 


\subsubsection{Disjunctive Weight Function}

Using a similar reasoning as in Section 2.2.1, Denœux [6] showed that any subnormal BBA $m$ can be uniquely decomposed as follows:

$$
m=\left(\left(\text { () }{ }_{A \supset \emptyset} A_{v_{C}(A)}\right)(\emptyset)\left((\mathrm{)}){ }_{A \supset \emptyset} A_{v_{D}(A)}\right),\right.
$$

where $v_{C}(A) \in(0,1], v_{D}(A) \in(0,1]$ and $\max \left(v_{C}(A), v_{D}(A)\right)=1$ for all $A \subseteq \Omega$ such that $A \neq \emptyset$, and the notation $A_{x}$ denotes the negation of $\bar{A}^{x}$. Let $v$ denote the mapping from $2^{\Omega} \backslash \emptyset$ to $(0,+\infty)$ defined as

$$
v(A)=\frac{v_{C}(A)}{v_{D}(A)}, \quad \forall A \supset \emptyset .
$$

This new function, referred to as the disjunctive weight function associated to $m$, may be recovered from $b$ as follows $[6]$ :

$$
\ln v(A)=-\sum_{B \subseteq A}(-1)^{|A|-|B|} \ln b(B), \quad \forall A \supset \emptyset .
$$

It is related to the conjunctive weight function $\bar{w}$ associated to the negation $\bar{m}$ of $m$ by the equation:

$$
v(A)=\bar{w}(\bar{A}), \quad \forall A \neq \emptyset .
$$

The TBM disjunctive rule has a simple expression using the disjunctive weight function. Let $m_{1}$ and $m_{2}$ be two subnormal BBAs with disjunctive weight functions $v_{1}$ and $v_{2}$, and let $v_{1 @ 2}$ be the disjunctive weight function associated to $m_{1}\left(m_{2}\right.$. We have:

$$
v_{1(0) 2}(A)=v_{1}(A) v_{2}(A), \quad \forall A \supset \emptyset
$$

\subsection{Informational Comparison of Belief Functions}

The least commitment principle (LCP) of the TBM postulates that, given a set of BBAs compatible with a set of constraints, the most appropriate BBA is the least informative [37]. It is similar to the principle of minimal specificity in possibility theory [45]. This principle becomes operational through the definition of partial orderings allowing the informational comparison of BBAs. Such orderings, generalizing set inclusion, were proposed by Yager [46] and Dubois and Prade [10]. Their interpretations are discussed from a set-theoretical perspective in [10] and from the point of view of the TBM in [12]. They are defined as follows:

- $p l$-ordering: $m_{1} \sqsubseteq_{p l} m_{2}$, iff $p l_{1}(A) \leq p l_{2}(A)$ for all $A \subseteq \Omega$;

- q-ordering: $m_{1} \sqsubseteq_{q} m_{2}$, iff $q_{1}(A) \leq q_{2}(A)$ for all $A \subseteq \Omega$; 
- $s$-ordering: $m_{1} \sqsubseteq_{s} m_{2}$, i.e., $m_{1}$ is a specialization of $m_{2}$, iff $m_{1}$ can be obtained from $m_{2}$ by transferring each mass $m_{2}(A)$ to subsets of $A$.

A BBA $m_{1}$ is said to be $x$-more committed than $m_{2}$, with $x \in\{p l, q, s\}$, if we have $m_{1} \sqsubseteq_{x} m_{2}$. It was shown in [10] that those definitions are not equivalent: $m_{1} \sqsubseteq_{s} m_{2}$ implies $m_{1} \sqsubseteq_{p l} m_{2}$ and $m_{1} \sqsubseteq_{q} m_{2}$, but the converse is not true. Furthermore, the orderings $\sqsubseteq_{p l}$ and $\sqsubseteq_{q}$ are not comparable. The vacuous BBA $m_{\Omega}$ is the unique greatest element for partial orderings $\sqsubseteq_{x}$ with $x \in\{s, q, p l\}$, i.e., we have $m \sqsubseteq_{x} m_{\Omega}$ for all $m$. Informally, this latter property means that all beliefs are more informative than ignorance, as should be.

In [6], Denœux defined a new partial ordering based on the conjunctive weight function. Given two nondogmatic BBAs $m_{1}$ and $m_{2}, m_{1}$ is said to be $w$-more committed, which is noted $m_{1} \sqsubseteq_{w} m_{2}$, iff $w_{1}(A) \leq w_{2}(A)$, for all $A \subset \Omega$. The vacuous BBA $m_{\Omega}$ is not a greatest element for $\sqsubseteq_{w}$, but it is only a maximal element (actually the unique maximal element), i.e., we have $m_{\Omega} \sqsubseteq_{w} m \Rightarrow m=m_{\Omega}$, for all nondogmatic bba $m$. It was shown in [6] that the $w$-ordering is strictly stronger than the $s$-ordering, i.e., we have, for any two nondogmatic BBAs $m_{1}$ and $m_{2}, m_{1} \sqsubseteq_{w} m_{2} \Rightarrow m_{1} \sqsubseteq_{s} m_{2}$.

Let us reproduce a lemma related to the $w$-ordering, which will be needed later.

Lemma 1 ([6]). Let $m$ be a nondogmatic BBA with conjunctive weight function $w$, and let $w^{\prime}$ be a mapping from $2^{\Omega} \backslash\{\Omega\}$ to $(0,+\infty)$ such that $w^{\prime}(A) \leq w(A)$ for all $A \subset \Omega$. Then $w^{\prime}$ is the conjunctive weight function of some nondogmatic BBA $\mathrm{m}^{\prime}$.

In [6], Denœux also defined yet another partial ordering, which is the disjunctive counterpart of $\sqsubseteq_{w}$. This ordering, called the $v$-ordering, is based on the disjunctive weight function. It is defined as follows: given two subnormal BBAs $m_{1}$ and $m_{2}, m_{1} \sqsubseteq v$ $m_{2}$ iff $v_{1}(A) \geq v_{2}(A)$ for all $A \neq \emptyset$. The $v$-ordering is strictly stronger than the $s$-ordering. However, $\sqsubseteq_{v}$ and $\sqsubseteq_{w}$ are not comparable.

As explained in [6], all these partial orderings seem equally well justified and reasonable. The choice of a particular partial ordering for a given problem is then guided by different considerations such as existence of a solution or tractability of calculations. In the following section, we review how the $\sqsubseteq w$ and $\sqsubseteq v$ orderings can be used to derive two rules of combination.

\subsection{Two Idempotent Rules Based on the Weight Functions}

We have seen that the TBM conjunctive and disjunctive rules are based on pointwise combination of conjunctive and disjunctive weights, respectively, using the product. Recently, Denœux [6] proposed two other rules, called the cautious and bold rules of 
combination, in which the product is replaced by the minimum. This section summarizes necessary material on those two rules.

\subsubsection{The Cautious Rule of Combination}

The TBM conjunctive rule is justified only when it is safe to assume that the items of evidence combined be distinct or, in other words, that the information sources be independent. When this assumption does not hold, an alternative consists in adopting a cautious, or conservative, attitude to the merging of belief functions by applying the LCP $[6,8,13]$. Let us now recall the building blocks of the cautious merging of belief functions.

As remarked in [10], it is possible to think of $\sqsubseteq_{x}$ as generalizing set inclusion. This reasoning can be used to see conjunctive combination rules as generalizing set intersection. Let us consider the following situation. Suppose we get two reliable sources of information. One states that $\omega$ is in $A \subseteq \Omega$, whereas the other states that it is in $B \subseteq \Omega$. It is then certain that $\omega$ is in $C$ such that $C \subseteq A$ and $C \subseteq B$. The largest subset $C$ satisfying those constraints is $A \cap B$. Now, suppose we get two reliable sources of information that provide two BBAs $m_{1}$ and $m_{2}$. Upon receiving those two pieces of information, the agent's state of belief should be represented by a BBA $m_{12}$ more informative than $m_{1}$ and $m_{2}$. Let $\mathcal{S}_{x}(m)$ be the set of BBAs $m^{\prime}$ such that $m^{\prime} \sqsubseteq_{x} m$, with $x \in\{v, w, s, q, p l\}$. Hence $m_{12} \in \mathcal{S}_{x}\left(m_{1}\right)$ and $m_{12} \in \mathcal{S}_{x}\left(m_{2}\right)$, or equivalently

$m_{12} \in \mathcal{S}_{x}\left(m_{1}\right) \cap \mathcal{S}_{x}\left(m_{2}\right)$. According to the LCP, the $x$-least committed BBA should be chosen in $\mathcal{S}_{x}\left(m_{1}\right) \cap \mathcal{S}_{x}\left(m_{2}\right)$. This defines a conjunctive combination rule if the $x$-least committed BBA exists and is unique. When $m_{1}$ and $m_{2}$ are nondogmatic, choosing the $w$-ordering yields an interesting solution [6, Proposition 4] which Denœux uses to define the so-called cautious rule of combination.

Definition $1([6])$. Let $m_{1}$ and $m_{2}$ be two nondogmatic $B B A s$, and let $m_{1} @ 2=m_{1} \oslash m_{2}$ denote the result of their combination by the cautious rule. The nondogmatic $B B A m_{1} \bowtie 2$ has the following conjunctive weight function:

$$
w_{1} \bigcap_{2}(A)=w_{1}(A) \wedge w_{2}(A), \quad \forall A \subset \Omega,
$$

where $\wedge$ is the minimum operator.

We thus have:

$$
m_{1} \bigcap_{2}=\bigcirc_{A \subset \Omega} A^{w_{1}(A) \wedge w_{2}(A)} .
$$

The cautious rule is commutative, associative, idempotent and monotonic with respect to $\sqsubseteq_{w}$. This last property means that if a nondogmatic BBA $m_{1}$ is less informative 
than a nondogmatic BBA $m_{2}$ according to the $\sqsubseteq_{w}$ ordering, then this order is unchanged after combination by $₫$ with a third nondogmatic BBA. Of interest for Section 4 of this paper is the fact that all of these properties are due to similar properties of the minimum on $(0,+\infty]$, much as the properties of the TBM conjunctive rule may be seen as consequences of the properties of the product on $(0,+\infty]$.

\subsubsection{The Bold Rule of Combination}

We have seen that the cautious rule of combination extends set intersection, and that it supposes the sources of information to be reliable. Let us now consider another situation. Suppose we get two sources of information and that it is known that at least one of the two sources is reliable, but we do not know which one. One of them states that $\omega$ is in $A \subseteq \Omega$, whereas the other one states that it is in $B \subseteq \Omega$. The smallest set containing both $A$ and $B$ is $A \cup B$. This reasoning is used in [6] to derive a disjunctive merging of belief functions based on the LCP, which can be summarized as follows. Suppose we get two sources of information that provide two BBAs $m_{1}$ and $m_{2}$, and that at least one of the sources is reliable but it is not known which one. Then, the BBA $m_{12}$ resulting from the merging of $m_{1}$ and $m_{2}$ should be the $x$-most committed BBA amongst the BBAs which are $x$-less committed than $m_{1}$ and $m_{2}$, with $x \in\{v, w, s, p l, q\}$ [6]. Denœux showed that when $m_{1}$ and $m_{2}$ are subnormal, using the $v$-ordering yields an interesting solution [6, Proposition 13], from which he defined the so-called bold rule of combination.

Definition 2 ([6]). Let $m_{1}$ and $m_{2}$ be two subnormal BBAs, and let $m_{1 @ 2}=m_{1} \otimes m_{2}$ denote the result of their combination by the bold rule. The disjunctive weight function of the subnormal $B B A m_{1 @ 2}$ is:

$$
v_{1 \otimes 2}(A)=v_{1}(A) \wedge v_{2}(A), \quad \forall A \neq \emptyset
$$

We thus have:

$$
m_{1 \otimes 2}=\left(_{A \neq \emptyset} A_{v_{1}(A) \wedge v_{2}(A)} .\right.
$$

The bold rule has similar properties as the cautious rule since they are both based on the minimum. Eventually, let us remark that the cautious and bold rules are related by De Morgan's laws [6]. We have:

$$
\begin{aligned}
& \overline{m_{1} \oslash m_{2}}=\overline{m_{1}} \oslash \overline{m_{2}}, \\
& \overline{m_{1} \otimes m_{2}}=\overline{m_{1}} \otimes \overline{m_{2}} .
\end{aligned}
$$




\section{Singularity of the TBM Conjunctive and Disjunctive Rules}

As shown above, the cautious and TBM conjunctive rules share a remarkable property: they are based on pointwise combination of conjunctive weights using a binary operator on $(0,+\infty]$. This binary operator is the minimum in the former case, and the product in the latter. As a consequence, the vacuous BBA is a neutral element for the TBM conjunctive rule, whereas it is not for the cautious rule. This property of the cautious rule seems difficult to interpret, as the vacuous BBA expresses total ignorance: as such, it could be expected to have no impact when combined conjunctively with any other BBA. We may thus wonder whether there exists a rule

1. based on pointwise combination of conjunctive weights (such a rule will be hereafter referred to as $w$-based);

2. having the vacuous BBA as neutral element, and

3. $w$-less committed than the TBM conjunctive rule.

As will be shown in Section 3.1, such a rule does not exist. We will demonstrate that the TBM conjunctive rule is less committed according the $\sqsubseteq_{w}$ ordering and, consequently, according to the orderings $\sqsubseteq_{x}$ with $x \in\{s, p l, q\}$ as well, than any other $w$-based rule having the vacuous BBA as neutral element. Section 3.2 will provide a counterpart to this result for the TBM disjunctive rule. Eventually, our justification of the TBM conjunctive rule will be compared in Section 3.3 to the other ones proposed in the literature.

\subsection{Justification of the TBM conjunctive rule}

Let us consider a $w$-based rule based on a binary operator $\circ$ on $(0,+\infty]$. It is clear that this rule has the vacuous BBA as neutral element if and only if 1 is a neutral element of $\circ$. We may remark that the product on $(0,+\infty]$ satisfies this property, whereas the minimum on $(0,+\infty]$ does not, hence the difference between the TBM conjunctive rule and the cautious rule.

However, not all binary operators on $(0,+\infty]$ correspond to a $w$-based rule, as the combination of two weight functions should yield a valid weight function associated to some BBA. The set of binary operators allowing the definition of a $w$-based rule with the vacuous BBA as neutral element is completely characterized by Proposition 1 and Theorem 1 below. We may already remark that this characterization is essential since it will lead to the justification of the TBM conjunctive rule. 
Proposition 1. Let $\circ$ be a binary operator on $(0,+\infty]$ such that $1 \circ x=x \circ 1=x$ for all $x \in(0,+\infty)$ and $x \circ y \leq x y$ for all $x, y \in(0,+\infty)$. Then, for any conjunctive weight functions $w_{1}$ and $w_{2}$, the function $w_{1 \circ 2}$ defined by:

$$
w_{1 \circ 2}(A)=w_{1}(A) \circ w_{2}(A), \quad \forall A \subset \Omega,
$$

is a conjunctive weight function associated to some nondogmatic $B B A m_{1 \circ 2}$.

Proof. We have

$$
w_{1 \circ 2}(A) \leq w_{1 @ 2}(A), \quad \forall A \subset \Omega .
$$

From Lemma $1, w_{1 \circ 2}$ is a conjunctive weight function since $w_{1 @ 2}$ is a conjunctive weight function.

Proposition 1 has shown that if a binary operator is below the product and has 1 as neutral element, then it can be used to define a $w$-based rule that has the vacuous BBA as neutral element. One may wonder if the constraint of being below the product can be relaxed. The answer is provided by the following theorem.

Theorem 1. Let $\circ$ be a binary operator on $(0,+\infty]$ such that

- $1 \circ x=x \circ 1=x$ for all $x \in(0,+\infty)$ and

- $x \circ y>x y$ for some $x, y \in(0,+\infty)$.

Then, there exist two nondogmatic $B B A s m_{1}$ and $m_{2}$ on a frame $\Omega$ such that the function obtained by pointwise combination using o of the conjunctive weight functions associated to $m_{1}$ and $m_{2}$ is not a conjunctive weight function.

Proof. See Appendix A.

The immediate corollary of this theorem constitutes one of the main results of this paper.

Corollary 1. The TBM conjunctive rule (a) is the $x$-least committed rule, with $x \in$ $\{w, s, p l, q\}$, among the $w$-based rules that have the vacuous $B B A m_{\Omega}$ as neutral element.

Proof. From Theorem 1 and Proposition 1, it is clear that any $w$-based rule $\odot$ that has the vacuous BBA as neutral element is based on a binary operator $\circ$ on $(0,+\infty]$ with 1 as neutral element and such that $x \circ y \leq x y$ for all $x, y \in(0,+\infty)$. For all nondogmatic BBAs $m_{1}$ and $m_{2}$, we thus have

$$
w_{1}(A) \circ w_{2}(A) \leq w_{1 @ 2}(A), \quad \forall A \subset \Omega .
$$

Consequently, $m_{1} \odot m_{2} \sqsubseteq_{w} m_{1} @ m_{2}$, and $m_{1} \odot m_{2} \sqsubseteq_{x} m_{1} @ m_{2}$ for $x \in\{s, p l, q\}$. 
According to this corollary, the TBM conjunctive rule thus respects a central principle of the TBM: the least commitment principle, under the two requirements of being based on pointwise combination of conjunctive weights and having the vacuous BBA as neutral element. Corollary 1 further implies that it is the only rule satisfying these properties. We thus have provided a new formal justification of the TBM conjunctive rule for combining nondogmatic belief functions.

In Section 3.3, this justification will be compared to the other ones proposed in the literature. Before that, we will first state a corresponding result for the TBM disjunctive rule, which is a consequence of the duality between these two rules.

\subsection{The Disjunctive Case}

The bold and TBM disjunctive rules are based on pointwise combination of disjunctive weights using a binary operator on $(0,+\infty]$ (respectively, the minimum and the product). The difference between these two rules is that the or-vacuous BBA $m_{\emptyset}$ is a neutral element for the TBM disjunctive rule, whereas it is not for the bold rule. This property is important in the context of a disjunctive merging. Indeed, it is a direct consequence of generalizing set union, as is the use of the principle of maximal commitment (instead of the LCP). The study of rules based on pointwise combination of disjunctive weights ( $v$-based rules for short) and which admits the or-vacuous BBA as neutral element leads to the following conclusion.

Corollary 2. The TBM disjunctive rule (1) is the $x$-most committed rule, with $x \in$ $\{v, s, p l, q\}$ among the $v$-based rules that have the or-vacuous BBA $m_{\emptyset}$ as neutral element.

Proof. Let $\circ$ be a binary operator on $(0,+\infty]$ having 1 as neutral element. Let $v_{1}$ and $v_{2}$ be the disjunctive weight functions associated to two subnormal BBAs $m_{1}$ and $m_{2}$. Let $\overline{w_{1}}$ and $\overline{w_{2}}$ be the conjunctive weight functions associated to $\overline{m_{1}}$ and $\overline{m_{2}}$. We have:

$$
\begin{aligned}
\overline{(4))_{A \neq \emptyset} A_{v_{1}(A) \circ v_{2}(A)}} & =\bigcirc_{A \neq \emptyset} \overline{A_{v_{1}(A) \circ v_{2}(A)}} \\
& =\bigcirc_{A \neq \emptyset} \bar{A}^{v_{1}(A) \circ v_{2}(A)}
\end{aligned}
$$

since $A_{x}$ denotes the negation of $\bar{A}^{x}$. Furthermore, we have from (5):

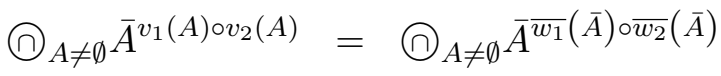

$$
\begin{aligned}
& =\bigcirc_{A \subset \Omega} A^{\overline{w_{1}}(A) \circ \overline{w_{2}}(A)} \text {. }
\end{aligned}
$$

From Theorem 1 and Lemma 1, (8) is guaranteed to be a BBA iff $\circ$ is such that $x \circ y \leq x y$. For any operator $\circ$ on $(0,+\infty]$ having 1 as neutral element and such that $x \circ y \leq x y$ for 
all $x, y \in(0,+\infty)$, we have

$$
v_{1}(A) \circ v_{2}(A) \leq v_{1(0) 2}(A), \quad \forall A \neq \emptyset .
$$

Consequently, the disjunctive rule based on $\circ$ is at most as $v$-committed as the rule (a). This result can be extended to weaker orderings $\sqsubseteq_{x}$ with $x \in\{s, p l, q\}$.

This corollary shows that the TBM disjunctive rule respects the principle of maximal commitment, which is the one to be followed in the context of disjunctive merging. It may thus be seen as a new justification for the TBM disjunctive rule.

\subsection{Discussion}

The justification of the TBM conjunctive rule proposed in this article as well as the ones proposed in $[9,36,18,19,16]$ completely fit the TBM since they are obtained without introducing any underlying probability concepts. However, it is interesting to remark that our approach is completely different from the other ones. Indeed, the justifications presented in $[36,18,19,16]$ result essentially from the associativity and commutativity properties required of the combination operator, as rightfully remarked by Smets in [44], and the justification of Dubois and Prade [9] is based on the requirement that the combination should satisfy the so-called separability property.

In comparison, our justification is based on two other requirements: the combination should be $w$-based and it should have the vacuous BBA as neutral element. Whereas the latter requirement is intuitively appealing, the former may seem more difficult to interpret. However, some justification may be found in the meaning of the canonical decomposition of a belief function, which breaks down a belief function into elementary pieces of evidence pertaining to single propositions. It may be argued that the combination of two belief functions should be performed by considering in turn each proposition and combining the two elementary pieces of evidence pertaining to it, which leads to the $w$-based requirement. As a further motivation for introducing this requirement, we may notice that $w$-based combinations offer a rarely considered, yet promising outlook on the combination of belief functions, as demonstrated by the recent introduction of the cautious rule.

As the arguments developed in this paper are based on the canonical conjunctive decomposition, which is rigorously defined only for nondogmatic belief functions, our justification of the TBM conjunctive rule is formally restricted to this particular class of belief functions. As noted in [39], it seems possible to extend this decomposition to any belief function using an infinitesimal discount rate. However, this idea remains to 
be fully investigated from a mathematical point of view. In any case, it may be argued that the restriction to nondogmatic belief functions is of little practical significance, as any belief function can always be discounted by an arbitrarily small amount, resulting in a more conservative representation of a piece of evidence [6].

In summary, all the justifications proposed for the TBM conjunctive rule seem reasonable and have their merits, even though the requirements on which they are based can always be subject to discussion. Globally, however, there seems to be a convergence of arguments in favor of this particular rule, even if other rules may be valuable in some situations as explained in the introduction of this paper. As a matter of fact, Section 4 below will reveal the existence of infinite families of combination rules in which the TBM conjunctive rule and the cautious rule are particular members.

\section{Four Infinite Families of Combination Rules}

As discussed in Section 1, having only one rule is not the ideal situation to cope with realworld problems. It could thus be useful to have other rules of combination. Such rules should at least satisfy a few basic properties such as commutativity or associativity. This section shows that the cautious and TBM conjunctive rules can be seen as particular members of two distinct families of combination rules. It thus provides an answer to the need for more flexibility in terms of combination rules, and sheds some new light on the fundamentally different behaviors of the cautious and TBM conjunctive rules. It also allows us to put the result of the preceding section in a broader perspective.

The key to the introduction of those families of rules is to remark that the cautious and TBM conjunctive rules are based on the minimum and the product, respectively, and that these two operators on $(0,+\infty]$ essentially differ by the position of their neutral element. Indeed, on the one hand, the minimum on $(0,+\infty)$ is commutative, associative, and monotonic. In addition, the upper bound of $(0,+\infty]$ serves as neutral element for the minimum. The minimum on $(0,+\infty]$ has thus similar properties as triangular norms (t-norms for short) [20], except that t-norms are usually defined on $[0,1]$. On the other hand, the product on $(0,+\infty]$ is commutative, associative, monotonic and has one as neutral element. It has thus similar properties as uninorms [47], which are usually defined as commutative, associative, monotonic operators on $[0,1]$ that admit a number $e \in[0,1]$ as neutral element. This comparison between the minimum and the product leads us to extend the definitions of t-norms and uninorms on $(0,+\infty]$ as follows.

Definition 3. A t-norm on $(0,+\infty]$ is a binary operator on $(0,+\infty]$, which is commutative, associative, monotonic, and which admits $+\infty$ as neutral element. 
Definition 4. A uninorm on $(0,+\infty]$ is a binary operator on $(0,+\infty]$, which is commutative, associative, monotonic, and which admits some positive real number $e \in(0,+\infty]$ as neutral element.

The construction of such generalized t-norms and generalized uninorms being out of the scope of this paper, the interested reader is referred to [26], where means are provided to obtain such operators out of t-norms on $[0,1]$. Example 1 below gives nonetheless an example of a t-norm on $(0,+\infty]$ different from the minimum and an example of a uninorm on $(0,+\infty]$ different from the product (the operators of Example 1 were obtained using the construction mechanisms provided in [26]).

Example 1. The operator $\star$ defined by

$$
x \star y= \begin{cases}\left(\frac{1}{x}+\frac{1}{y}-\frac{1}{x} \cdot \frac{1}{y}\right)^{-1} & \text { if } x \wedge y>1 \\ x \wedge y & \text { otherwise }\end{cases}
$$

for all $x, y \in(0,+\infty]$ is a $t$-norm on $(0,+\infty]$.

The operator $\circ$ defined by

$$
x \circ y= \begin{cases}x \cdot y & \text { if } x \vee y \leq 1 \\ ((1 / x) \wedge(1 / y))^{-1} & \text { if } x \wedge y \geq 1 \\ x \wedge y & \text { otherwise, }\end{cases}
$$

for all $x, y \in(0,+\infty]$ is a uninorm on $(0,+\infty]$ having 1 as neutral element. We may further remark that this uninorm verifies $x \circ y \leq x y$ for all $x, y \in(0,+\infty]$.

\subsection{Conjunctive T-rules}

As previously mentioned, the minimum is a t-norm on $(0,+\infty]$. The cautious rule thus belongs to a family of rules based on pointwise combination of conjunctive weights using t-norms on $(0,+\infty]$. In order to characterize this family, we need to remark that the minimum is the largest t-norm on $(0,+\infty]$, much as it is the largest t-norm on $[0,1]$.

Lemma 2. The minimum is the largest $t$-norm on $(0,+\infty]$.

Proof. Any t-norm $\star$ on $(0,+\infty]$ has by definition $+\infty$ as neutral element and is monotonic, hence we have $x \star y \leq x \star+\infty=x$ and $x \star y \leq+\infty \star y=y$, so $x \star y \leq x \wedge y$, for all $x, y \in(0,+\infty]$.

We may then show the following. 
Proposition 2. Let $\star$ be a t-norm on $(0,+\infty]$. Then, for any conjunctive weight functions $w_{1}$ and $w_{2}$, the function $w_{1 \circledast w_{w} 2}$ defined by:

$$
w_{1 \circledast{ }_{w}^{2}}(A)=w_{1}(A) \star w_{2}(A), \quad \forall A \subset \Omega,
$$

is a conjunctive weight function associated to some nondogmatic $B B A m_{1 \circledast_{w} 2}$.

Proof. From Lemma 2, we have

$$
w_{1 \circledast{ }_{w}}(A) \leq w_{1} \circledast_{2}(A), \quad \forall A \subset \Omega .
$$

From Lemma $1, w_{1 \circledast_{w}} 2$ is a conjunctive weight function since $w_{1} \oslash_{2}$ is a conjunctive weight function.

Proposition 2 allows us to define combination rules for nondogmatic belief functions which can be formally defined as follows.

Definition 5 (T-norm-based conjunctive combination rule). Let $\star$ be a t-norm on $(0,+\infty]$. Let $m_{1}$ and $m_{2}$ be two nondogmatic BBAs. Their combination using the t-norm-based conjunctive combination rule, or conjunctive t-rule for short, is noted $m_{1 \circledast_{w} 2}=m_{1} \circledast_{w} m_{2}$. It is defined as a nondogmatic BBA with the following conjunctive weight function:

$$
w_{1 \circledast{ }_{w}{ }^{2}}(A)=w_{1}(A) \star w_{2}(A), \quad \forall A \subset \Omega .
$$

We thus have:

$$
m_{1 \circledast{ }_{w} 2}=\bigcirc_{A \subset \Omega} A^{w_{1}(A) \star w_{2}(A)} .
$$

Proposition 3. Any conjunctive t-rule $\circledast w$ has the following properties:

- Commutativity: for all nondogmatic BBAs $m_{1}$ and $m_{2}, m_{1} \circledast{ }_{w} m_{2}=m_{2} \circledast w_{w} m_{1}$;

- Associativity: for all nondogmatic BBAs $m_{1}, m_{2}$ and $m_{3}$,

$$
m_{1} \circledast{ }_{w}\left(m_{2} \circledast{ }_{w} m_{3}\right)=\left(m_{1} \circledast{ }_{w} m_{2}\right) \circledast{ }_{w} m_{3} ;
$$

- Monotonicity with respect to $\sqsubseteq_{w}$ : for all nondogmatic $B B A s m_{1}, m_{2}$ and $m_{3}$, we have $m_{1} \sqsubseteq_{w} m_{2} \Rightarrow m_{1} \circledast_{w} m_{3} \sqsubseteq_{w} m_{2} \circledast{ }_{w} m_{3}$;

Proof. These properties follow directly from corresponding properties of the t-norm $\star$.

Finally, the following proposition situates the cautious rule in the family of conjunctive t-rules. 
Proposition 4. Among all conjunctive t-rules, the cautious rule is the $x$-least committed, with $x \in\{w, s, p l, q\}$ :

$$
m_{1} \circledast{ }_{w} m_{2} \quad \sqsubseteq_{x} \quad m_{1} \circledast m_{2},
$$

for all nondogmatic BBAs $m_{1}$ and $m_{2}$.

Proof. Since the minimum is the largest t-norm on $(0,+\infty]$, we have, for all nondogmatic BBAs $m_{1}$ and $m_{2}, m_{1} \circledast{ }_{w} m_{2} \sqsubseteq_{w} m_{1} \oslash m_{2}$, and $m_{1} \circledast{ }_{w} m_{2} \sqsubseteq_{x} m_{1} @ m_{2}$ for $x \in\{s, p l, q\}$.

\subsection{Conjunctive U-rules}

We have seen that the TBM conjunctive rule is based on the product and that the product is a uninorm on $(0,+\infty]$ with 1 as neutral element (1-uninorm for short). Hence, the TBM conjunctive rule belongs to a family of rules characterized by pointwise combination of conjunctive weights using 1-uninorms. From Proposition 1 and Theorem 1, the condition that those uninorms must respect is known, i.e., they must satisfy $x \circ y \leq x y$ for all $x, y \in(0,+\infty)$. New combination rules based on uninorms may thus be defined as follows.

Definition 6 (Uninorm-based conjunctive combination rule). Let o be a 1-uninorm, such that $x \circ y \leq x y$ for all $x, y \in(0,+\infty)$. Let $m_{1}$ and $m_{2}$ be two nondogmatic BBAs. Their combination using the uninorm-based conjunctive combination rule, or conjunctive u-rule for short, is noted $m_{1 \bigodot_{w} 2}=m_{1} \bigodot_{w} m_{2}$. It is defined as a nondogmatic BBA with the following conjunctive weight function:

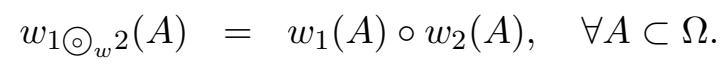

We thus have:

$$
m_{1 \bigodot_{w} 2}=\bigcirc_{A \subset \Omega} A^{w_{1}(A) \circ w_{2}(A)} .
$$

Proposition 5. Any conjunctive u-rule $\odot_{w}$ is commutative, associative, monotonic with respect to $\sqsubseteq_{w}$, and such that: $m \bigodot_{w} m_{\Omega}=m$, for all nondogmatic BBA $m$.

Proof. These properties follow directly from corresponding properties of the uninorm ○.

The next proposition shows that the TBM conjunctive rule has a special position in the family of the conjunctive u-rules. 
Proposition 6. Among all conjunctive u-rules, the TBM conjunctive rule is the $x$-least committed, with $x \in\{w, s, p l, q\}$ :

$$
m_{1} \bigodot_{w} m_{2} \quad{ }_{x} \quad m_{1} @ m_{2},
$$

for all nondogmatic BBAs $m_{1}$ and $m_{2}$.

Proof. From the definition of the conjunctive u-rules, we have, for all nondogmatic BBAs $m_{1}$ and $m_{2}, m_{1} \bigcirc_{w} m_{2} \sqsubseteq_{w} m_{1} @ m_{2}$, and $m_{1} \bigcirc_{w} m_{2} \sqsubseteq_{x} m_{1} @ m_{2}$ for $x \in\{s, p l, q\}$.

Finally, we complete the characterization of the conjunctive u-rules by the following proposition.

Proposition 7. Conjunctive u-rules are not idempotent.

Proof. This follows from the fact that idempotence and having the vacuous BBA as neutral element are incompatible properties for $w$-based rules. Indeed, from Proposition 1 and Theorem 1, a $w$-based rule that has the vacuous BBA as neutral element is based on a binary operator $\circ$ satisfying $x \circ y \leq x y$, for all $x, y>0$. Let $z \in(0,1)$, we have $z \circ z \leq z^{2}<z$, hence $\circ$ is not idempotent.

\subsection{Disjunctive T-rules and U-rules}

For the sake of completeness, this section presents results corresponding to the previous ones for $v$-based rules. Obvious results are stated succinctly, whereas De Morgan relations between conjunctive and disjunctive rules are more detailed.

\subsubsection{Disjunctive T-rules}

The bold rule is based on the minimum. Hence, it belongs to a family of rules based on pointwise combination of disjunctive weights using t-norms on $(0,+\infty]$. The counterpart of Proposition 2 for disjunctive weights allows us to define a belief function combination rule $\circledast v$, called a disjunctive t-rule, as

$$
m_{1 \circledast_{v} 2}=\left(_{A \neq \emptyset} A_{v_{1}(A) \star v_{2}(A)},\right.
$$

where $\star$ is a t-norm on $(0,+\infty]$, and $m_{1}$ and $m_{2}$ are two subnormal BBAs.

Any disjunctive t-rule $\circledast_{v}$ is commutative, associative and monotonic with respect to $\sqsubseteq_{v}$. Furthermore, it may easily be shown, using similar arguments as developed in Section 4.1, that the bold rule is the $x$-most committed disjunctive t-rule, with $x \in$ $\{v, s, p l, q\}$. 
Finally, the following proposition shows that the $\circledast w$ and $\circledast v$ operations are dual to each other with respect to complementation, i.e., they are linked by De Morgan laws analogous to (6) and (7).

Proposition 8. Let $\circledast w$ and $\circledast v$ be respectively, conjunctive and disjunctive $t$-rules based on a $t$-norm $\star$ on $(0,+\infty]$. We have:

$$
\overline{m_{1} \circledast{ }_{v} m_{2}}=\overline{m_{1}} \circledast{ }_{w} \overline{m_{2}},
$$

for all subnormal BBAs $m_{1}$ and $m_{2}$, and

$$
\overline{\left.m_{1} \circledast\right)_{w} m_{2}}=\overline{m_{1} \circledast v} \overline{m_{2}}
$$

for all nondogmatic BBAs $m_{1}$ and $m_{2}$.

Proof. Let $m_{1}$ and $m_{2}$ be two subnormal BBAs. We have

$$
\begin{aligned}
\overline{m_{1} \circledast{ }_{v} m_{2}} & =\overline{(\cup)_{A \neq \emptyset} A_{v_{1}(A) \star v_{2}(A)}} \\
& =\bigcirc_{A \neq \emptyset} \overline{A_{v_{1}(A) \star v_{2}(A)}} \\
& =\bigcirc_{A \neq \emptyset} \bar{A}^{\overline{w_{1}}(\bar{A}) \star \overline{w_{2}}}(\bar{A}) \\
& =\bigcirc_{A \subset \Omega} A^{\overline{w_{1}}(A) \star \overline{w_{2}}}(A) \\
& =\overline{m_{1}} \circledast_{w} \overline{m_{2}} .
\end{aligned}
$$

The proof of (9) is similar.

\subsubsection{Disjunctive U-rules}

The TBM disjunctive rule is based on the product of disjunctive weights. Hence, it belongs to a family of rules defined by pointwise combination of disjunctive weights using 1-uninorms. From Corollary 2, the condition that those uninorms must respect is known. We may thus define a belief function combination rule $\odot_{v}$, called a disjunctive u-rule, as

$$
m_{1 \bigodot_{v} 2}=\bigodot_{A \neq \emptyset} A_{v_{1}(A) \circ v_{2}(A)},
$$

where $\circ$ is a 1 -uninorm, such that $x \circ y \leq x y$ for all $x, y \in(0,+\infty)$, and where $m_{1}$ and $m_{2}$ are two subnormal BBAs.

Any disjunctive u-rule $\bigodot_{v}$ is commutative, associative, monotonic with respect to $\sqsubseteq_{v}$ and has the BBA $m_{\emptyset}$ as neutral element. Furthermore, the TBM disjunctive rule is the $x$-most committed disjunctive u-rule, with $x \in\{v, s, p l, q\}$.

Finally, the following proposition shows that the $\bigodot_{w}$ and $\bigodot_{v}$ operations are dual to each other with respect to complementation. 
Proposition 9. Let $\odot_{w}$ and $\odot_{v}$ be respectively, conjunctive and disjunctive u-rules based on a 1-uninorm $\circ$. We have:

$$
\overline{m_{1} \odot{ }_{v} m_{2}}=\overline{m_{1}} \bigodot_{w} \overline{m_{2}},
$$

for all subnormal $B B A s m_{1}$ and $m_{2}$, and

$$
\overline{m_{1} \bigodot_{w} m_{2}}=\overline{m_{1}} \bigodot_{v} \overline{m_{2}},
$$

for all nondogmatic BBAs $m_{1}$ and $m_{2}$.

Proof. The proof of (10) is direct using the proof of Proposition 2. The proof of (11) is similar.

\section{Conclusion}

In this paper, it has been shown that the unnormalized Dempster's rule of combination is the least committed rule among the rules based on pointwise combination of conjunctive weights and that have the vacuous belief function as neutral element. This constitutes a new justification for this rule as a mechanism for combining distinct and nondogmatic belief functions.

It has also been brought forward that the unnormalized Dempster's rule $($ ) and the more recent cautious rule $₫$ have fundamental different algebraic properties: the former is based on a uninorm on $(0,+\infty]$ and has a neutral element while the latter is based on a t-norm on $(0,+\infty]$ and has no neutral element. Similar properties hold for the disjunctive duals of these two rules, namely the TBM disjunctive rule (5) and the bold rule $(\nabla$.

In addition, it was revealed that to each of those four basic rules corresponds one infinite family of combination rules. Indeed, there exist two t-norm-based families that are based, respectively, on the conjunctive and disjunctive weight functions. There exist also two uninorm-based families that are based, respectively, on the conjunctive and disjunctive weight functions. It was also shown that t-norm-based conjunctive and disjunctive rules, as well as uninorm-based conjunctive and disjunctive rules, are related by De Morgan laws. The existence of such families of rules suggests that DempsterShafer theory is not poorer than Possibility theory [11] in terms of fusion operators, as already noted in [6].

Of particular interest is that the four basic rules occupy a special position in each of their respective family: the $(a)$ and $₫$ rules are the least committed elements, whereas the (a) and (1) rules are the most committed elements. This is summarized in Figure 1. 


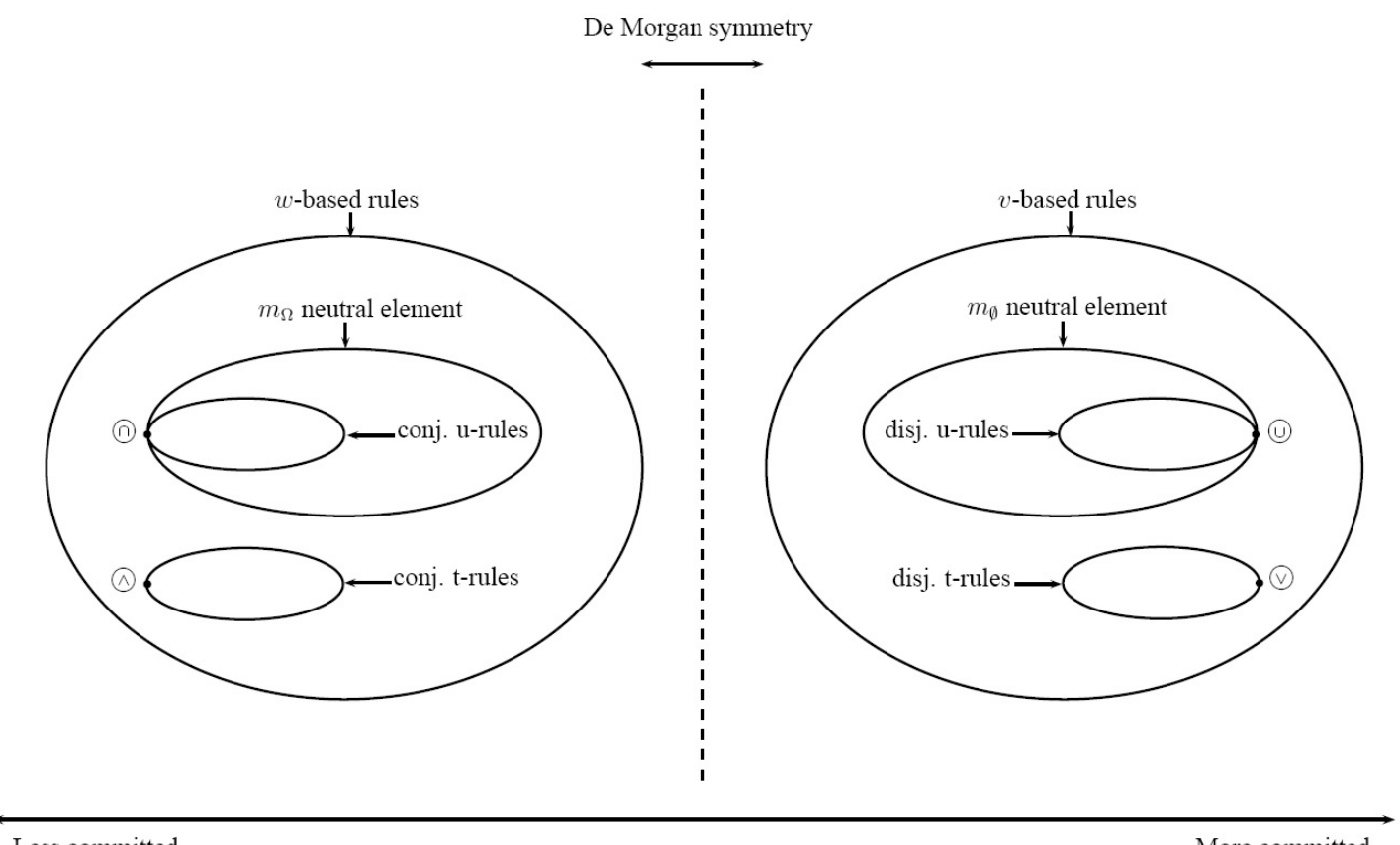

Less committed

More committed

Figure 1: The four families of combination rules studied in this paper, and the singular positions of the four basic rules $(1),($, (๑) and $($ )

To conclude, it is worth mentioning that, despite the numerous properties shared by the unnormalized Dempster's rule and the conjunctive u-rules, it was shown in [27] that the unnormalized Dempster's rule is the only conjunctive u-rule that satisfies a particular axiom of the valuation algebra framework [21]. This property further singles out this rule among uninorm-based conjunctive combination rules.

\section{Acknowledgments}

The authors would like to thank the anonymous referees of the special track Uncertain Reasoning of the FLAIRS'08 conference, as well as the anonymous referees of this special issue on Uncertain Reasoning of the Journal of Automated Reasoning, for their constructive comments that helped us to improve this paper.

\section{A Proof of Theorem 1}

The proof of Theorem 1 requires the two following technical lemmas (Lemmas 3 and 4). 
Lemma 3. Let $m$ be a BBA. For $B \subset \Omega$, the following equality holds:

$$
\sum_{A \subseteq B}(-1)^{|A|} q(A)=\sum_{A \cap B=\emptyset} m(A)
$$

Proof. Let $m_{B}$ denote a BBA with single focal element $B \subset \Omega$. Let $m$ be a BBA and $m^{\prime}=m @ m_{B}$. We have

$$
m^{\prime}(\emptyset)=\sum_{A \cap B=\emptyset} m(A)
$$

Let $q_{B}$ denote the commonality function associated to $m_{B}$.

$$
q_{B}(A)= \begin{cases}1 & \text { if } A \subseteq B \\ 0 & \text { otherwise }\end{cases}
$$

Let $q^{\prime}$ and $q$ denote the commonality functions associated to $m^{\prime}$ and $m$, respectively. We have:

$$
q^{\prime}(A)=q(A) \cdot q_{B}(A) \quad \forall A \subseteq \Omega
$$

Hence

$$
q^{\prime}(A)= \begin{cases}q(A) & \text { if } A \subseteq B \\ 0 & \text { otherwise. }\end{cases}
$$

Consequently, using (2), we have

$$
\begin{aligned}
m^{\prime}(\emptyset) & =\sum_{C \subseteq \Omega}(-1)^{|C|} q^{\prime}(C) \\
& =\sum_{A \subseteq B}(-1)^{|A|} q(A),
\end{aligned}
$$

which completes the proof.

Lemma 4. Let $m$ be a normal, nondogmatic BBA and such that $m(C)>0$, for a proper subset $C \subset \Omega$. Let $w$ be the conjunctive weight function associated to $m$. Further, let $m^{\prime}=m @ B^{w(B)} @ B^{w(B)+\epsilon}$, with $B \subset \Omega, C \cap B=\emptyset$ and $\epsilon>0 . m^{\prime}$ is not a $B B A$.

Proof. The proof consists in showing that $m^{\prime}(\emptyset)<0$. Let $B$ be a strict subset of $\Omega$ such that $C \cap B=\emptyset$. The following equality holds:

$$
m^{\prime}(\emptyset)=\sum_{A \subseteq \Omega}(-1)^{|A|} \frac{q(A)}{q_{B}(A)} q_{B}^{\prime}(A),
$$


where $q, q_{B}$ and $q_{B}^{\prime}$ are the commonality functions associated to $m, B^{w(B)}$ and $B^{w(B)+\epsilon}$, respectively. We have:

$$
\begin{gathered}
q_{B}(A)= \begin{cases}1 & \text { if } A \subseteq B, \\
w(B) & \text { otherwise, }\end{cases} \\
q_{B}^{\prime}(A)= \begin{cases}1 & \text { if } A \subseteq B, \\
w(B)+\epsilon & \text { otherwise }\end{cases}
\end{gathered}
$$

Using (12) and (13), one can obtain:

$$
m^{\prime}(\emptyset)=\sum_{A \subseteq B}(-1)^{|A|} q(A)+\sum_{A \nsubseteq B}(-1)^{|A|} q(A) \frac{w(B)+\epsilon}{w(B)} .
$$

As

$$
\begin{aligned}
m(\emptyset) & =\sum_{A \subseteq \Omega}(-1)^{|A|} q(A) \\
& =\sum_{A \subseteq B}(-1)^{|A|} q(A)+\sum_{A \nsubseteq B}(-1)^{|A|} q(A),
\end{aligned}
$$

then

$$
m^{\prime}(\emptyset)=m(\emptyset)+\frac{\epsilon}{w(B)} \sum_{A \nsubseteq B}(-1)^{|A|} q(A) .
$$

We can thus remark that $m^{\prime}(\emptyset)$ is equal to $m(\emptyset)$, which is itself equal to 0 , plus another term. Let us prove that this term is always strictly smaller than 0 . We have

$$
\begin{aligned}
\frac{\epsilon}{w(B)} \sum_{A \nsubseteq B}(-1)^{|A|} q(A) & =\frac{\epsilon}{w(B)}\left(m(\emptyset)-\sum_{A \subseteq B}(-1)^{|A|} q(A)\right) \\
& =-\frac{\epsilon}{w(B)} \sum_{A \subseteq B}(-1)^{|A|} q(A) .
\end{aligned}
$$

We thus have from Lemma 3:

$$
m^{\prime}(\emptyset)=-\frac{\epsilon}{w(B)} \sum_{A \cap B=\emptyset} m(A) .
$$

As $m(C)>0$ for $C$ such that $C \cap B=\emptyset$, the sum in the right-hand side of (14) is strictly greater than zero. Further we have $\epsilon>0$ and $w(B)>0$. Hence $m^{\prime}(\emptyset)<0$, thus $m^{\prime}$ is not a BBA.

Theorem 1 can then be proved as follows.

Proof. Let $x$ and $y$ be any two numbers such that $x \circ y>x y$. Obviously, as 1 is assumed to be a neutral element of $\circ$, we have $x \neq 1$ and $y \neq 1$. Let $\epsilon=x \circ y-x y>0$. The proof consists in choosing two logically consistent BBAs $m_{1}$ and $m_{2}$, i.e., $m_{1 @ 2}(\emptyset)=0$, such that: 
- $\exists B \in 2^{\Omega} \backslash\{\Omega\}$ such that $w_{1}(B)=x$ and $w_{2}(B)=y$;

- $\forall A \in 2^{\Omega} \backslash\{\Omega, B\}, w_{1}(A)=1$ or $w_{2}(A)=1$;

- $\exists C \in 2^{\Omega}$ such that $m_{1 @ 2}(C)>0$ and $C \cap B=\emptyset$.

For those BBAs, we thus have:

$$
\begin{aligned}
& w_{1 @ 2}(B)=w_{1}(B) \cdot w_{2}(B), \\
& w_{1 @ 2}(A)= \begin{cases}w_{1}(A) & \text { if } w_{2}(A)=1, \\
w_{2}(A) & \text { otherwise, }\end{cases}
\end{aligned}
$$

for all $A \neq B$, and

$$
\begin{aligned}
& w_{1}(B) \circ w_{2}(B)=w_{1 @ 2}(B)+\epsilon, \\
& w_{1}(A) \circ w_{2}(A)=w_{1 @ 2}(A),
\end{aligned}
$$

for all $A \neq B$. Hence, we have:

$$
\begin{aligned}
& (\cap)_{A \subset \Omega} A^{w_{1}(A) \circ w_{2}(A)}=()_{A \subset \Omega,} A^{w_{1} @_{2}(A)} @ B^{w_{1} @ 2(B)+\epsilon} \\
& A \neq B \\
& =\bigcirc_{A \subset \Omega} A^{w_{1} @ 2(A)} \oslash B^{w_{1} @{ }_{2}(B)} @ B^{w_{1} @ 2(B)+\epsilon} \\
& =m_{1 @ 2} @ B^{w_{1} @ 2(B)} @ B^{w_{1} @ 2(B)+\epsilon} \text {, }
\end{aligned}
$$

and $\exists C \in 2^{\Omega}$ such that $m_{1 @ 2}(C)>0$ and $C \cap B=\emptyset$. By Lemma $4,(15)$ is not a BBA, hence $w_{1} \circ w_{2}$ is not a conjunctive weight function of some nondogmatic BBA.

Let us now provide the BBAs $m_{1}$ and $m_{2}$ which verify the above scheme. Since the considered numbers $x$ and $y$ take their values in $(0,+\infty) \backslash\{1\}$, we consider in the remainder of this proof the following cases:

- Case 1: $x \vee y<1$;

- Case 2: $x \wedge y>1$;

- Case 3: $x \vee y>1$ and $x \wedge y<1$.

We must thus provide a pair of BBAs $m_{1}$ and $m_{2}$ verifying the above scheme for each of the three possible cases.

- Case 1: 
Let $\Omega=\{a, b, c\}$ and let $m_{1}$ and $m_{2}$ be two BBAs defined on $\Omega$ as follows, for $\alpha, \beta \in(0,0.5)$ :

$$
\begin{aligned}
& m_{1}(A)= \begin{cases}\alpha & \text { if } A=\{a, b\} \text { or } A=\{b, c\}, \\
1-2 \alpha & \text { if } A=\Omega, \\
0 & \text { otherwise. }\end{cases} \\
& m_{2}(A)= \begin{cases}\beta & \text { if } A=\{a, c\} \text { or } A=\{b, c\}, \\
1-2 \beta & \text { if } A=\Omega, \\
0 & \text { otherwise. }\end{cases}
\end{aligned}
$$

The conjunctive weight functions associated to those BBAs are:

$$
\begin{aligned}
& w_{1}(A)= \begin{cases}\frac{(1-2 \alpha)}{(1-\alpha)} & \text { if } A=\{a, b\} \text { or } A=\{b, c\}, \\
\frac{(1-\alpha)^{2}}{(1-2 \alpha)} & \text { if } A=\{b\} \\
1 & \text { otherwise. }\end{cases} \\
& w_{2}(A)= \begin{cases}\frac{(1-2 \beta)}{(1-\beta)} & \text { if } A=\{a, c\} \text { or } A=\{b, c\}, \\
\frac{(1-\beta)^{2}}{(1-2 \beta)} & \text { if } A=\{c\}, \\
1 & \text { otherwise. }\end{cases}
\end{aligned}
$$

For those two BBAs, we have:

$-m_{1 @ 2}(\emptyset)=0$,

- $\exists B=\{b, c\}$ such that $w_{1}(B)=x, x \in(0,1)$ as $w_{1}(B)=f(\alpha)$ with $f$ a surjective function from $(0,0.5)$ to $(0,1)$, and $w_{2}(B)=y, y \in(0,1)$, as $w_{2}(B)=g(\beta)$ with $g$ a surjective function from $(0,0.5)$ to $(0,1)$.

- $\forall A \in 2^{\Omega} \backslash\{B, \Omega\}, w_{1}(A)=1$ or $w_{2}(A)=1$,

- $\exists C=\{a\}$ such that $m_{1 @ 2}(C)>0$ and $C \cap B=\emptyset$.

- Case 2:

Let $\Omega=\{a, b, c, d, e\}$ and let $m_{1}$ and $m_{2}$ be two BBAs defined on $\Omega$ as follows, for $\alpha \in(0,0.5)$ and $\beta \in(0,1 / 3)$ :

$$
m_{1}(A)= \begin{cases}\alpha & \text { if } A=\{a, b\} \text { or } A=\{b, c\} \\ 1-2 \alpha & \text { if } A=\Omega \\ 0 & \text { otherwise }\end{cases}
$$




$$
m_{2}(A)= \begin{cases}\beta & \text { if } A \in\{\{a, b, c\},\{a, c, e\},\{b, d, e\}\} \\ 1-3 \beta & \text { if } A=\Omega \\ 0 & \text { otherwise. }\end{cases}
$$

The conjunctive weight functions associated to those BBAs are:

$$
\begin{gathered}
w_{1}(A)= \begin{cases}\frac{(1-2 \alpha)}{(1-\alpha)} & \text { if } A=\{a, b\} \text { or } A=\{b, c\} \\
\frac{(1-\alpha)^{2}}{(1-2 \alpha)} & \text { if } A=\{b\} \\
1 & \text { otherwise. }\end{cases} \\
w_{2}(A)= \begin{cases}\frac{1-3 \beta}{1-2 \beta} & \text { if } A \in\{\{a, b, c\},\{a, c, e\},\{b, d, e\}\}, \\
\frac{(1-\beta)^{3}(1-3 \beta)}{(1-2 \beta)^{3}} & \text { if } A=\{\emptyset\}, \\
\frac{(1-2 \beta)^{2}}{(1-\beta)(1-3 \beta)} & \text { if } A \in\{\{b\},\{e\},\{a, c\}\}, \\
1 & \text { otherwise. }\end{cases}
\end{gathered}
$$

For those two BBAs, we have:

$-m_{1 @ 2}(\emptyset)=0$,

$-\exists B=\{b\}$ such that $w_{1}(B)=x, x \in(1,+\infty)$ as $w_{1}(B)=f(\alpha)$ with $f$ a surjective function from $(0,0.5)$ to $(1,+\infty)$, and $w_{2}(B)=y, y \in(1,+\infty)$, as $w_{2}(B)=g(\beta)$ with $g$ a surjective function from $(0,1 / 3)$ to $(1,+\infty)$.

- $\forall A \in 2^{\Omega} \backslash\{B, \Omega\}, w_{1}(A)=1$ or $w_{2}(A)=1$,

- $\exists C=\{a\}$ such that $m_{1 @ 2}(C)>0$ and $C \cap B=\emptyset$.

- Case 3:

Let $\Omega=\{a, b, c, d\}$ and let $m_{1}$ and $m_{2}$ be two BBAs defined on $\Omega$ as follows, for $\alpha \in(0,0.5)$ and $\beta \in(0,1 / 3)$ :

$$
\begin{gathered}
m_{1}(A)= \begin{cases}\alpha & \text { if } A=\{a, b\} \text { or } A=\{b, c\}, \\
1-2 \alpha & \text { if } A=\Omega, \\
0 & \text { otherwise. }\end{cases} \\
m_{2}(A)= \begin{cases}\beta & \text { if } A \in\{\{a, b, c\},\{a, b, d\},\{a, c, d\}\}, \\
1-3 \beta & \text { if } A=\Omega, \\
0 & \text { otherwise. }\end{cases}
\end{gathered}
$$

The conjunctive weight functions associated to those BBAs are:

$$
w_{1}(A)= \begin{cases}\frac{(1-2 \alpha)}{(1-\alpha)} & \text { if } A=\{a, b\} \text { or } A=\{b, c\} \\ \frac{(1-\alpha)^{2}}{(1-2 \alpha)} & \text { if } A=\{b\} \\ 1 & \text { otherwise. }\end{cases}
$$




$$
w_{2}(A)= \begin{cases}\frac{1-3 \beta}{1-2 \beta} & \text { if } A \in\{\{a, b, c\},\{a, b, d\},\{a, c, d\}\} \\ \frac{(1-\beta)^{3}(1-3 \beta)}{(1-2 \beta)^{3}} & \text { if } A=\{a\} \\ \frac{(1-2 \beta)^{2}}{(1-\beta)(1-3 \beta)} & \text { if } A \in\{\{a, b\},\{a, c\},\{a, d\}\} \\ 1 & \text { otherwise. }\end{cases}
$$

For those two BBAs, we have:

$-m_{1 \bigcirc 2}(\emptyset)=0$,

- $\exists B=\{a, b\}$ such that $w_{1}(B)=x, x \in(0,1)$ as $w_{1}(B)=f(\alpha)$ with $f$ a surjective function from $(0,0.5)$ to $(0,1)$, and $w_{2}(B)=y, y \in(1,+\infty)$, as $w_{2}(B)=g(\beta)$ with $g$ a surjective function from $(0,1 / 3)$ to $(1,+\infty)$.

- $\forall A \in 2^{\Omega} \backslash\{B, \Omega\}, w_{1}(A)=1$ or $w_{2}(A)=1$,

- $\exists C=\{c\}$ such that $m_{1 @ 2}(C)>0$ and $C \cap B=\emptyset$.

\section{References}

[1] Ayoun, A., Smets, Ph.: Data association in multi-target detection using the transferable belief model. Int. J. Intell. Syst. 10(16), 1167-1182 (2001)

[2] Benavoli, A., Chisci, L., Ristic, B., Farina, A., Graziano, A.: Reasoning under uncertainty: from Bayes to Valuation Based Systems. Application to target classification and threat evaluation. Selex Sistemi Integrati, Università degli Studi di Firenze, Italy (2007)

[3] Cattaneo, M. E. G. V.: Combining belief functions issued from dependent sources. In: Bernard, J. M., Seidenfeld, T., Zaffalon, M. (eds.) Proceedings of the Third International Symposium on Imprecise Probabilities and Their Applications (ISIPTA’03), Lugano, Switzerland, 2003, pp 133-147. Carleton Scientific (2003)

[4] Dempster, A. P.: Upper and lower probabilities induced by a multivalued mapping. Ann. Math. Statistics 38, 325-339 (1967)

[5] Denœux, T.: A neural network classifier based on Dempster-Shafer theory. IEEE transactions on Systems, Man and Cybernetics A 30(2), 131-150 (2000)

[6] Denœux, T.: Conjunctive and Disjunctive Combination of Belief Functions Induced by Non Distinct Bodies of Evidence. Artificial Intelligence 172, 234-264 (2008) 
[7] Denœux, T., Smets, Ph.: Classification using Belief Functions: the Relationship between the Case-based and Model-based Approaches. IEEE Transactions on Systems, Man and Cybernetics B 36(6), 1395-1406 (2006)

[8] Destercke, S., Dubois, D., Chojnacki, E.: Cautious Conjunctive Merging of Belief Functions. In: Mellouli, K. (ed.) Proceedings of the 9th European Conference on Symbolic and Quantitative Approaches to Reasoning with Uncertainty (ECSQARU'07), Hammamet, Tunisia, 2007. Lecture Notes in Computer Science. Lecture Note in Artificial Intelligence, vol. 4724, pp 332-343. Springer-Verlag (2007)

[9] Dubois, D., Prade, H.: On the Unicity of Dempster Rule of Combination. Int. J. Intell. Syst. 1, 133-142 (1986)

[10] Dubois, D., Prade, H.: A set-theoretic view of belief functions: logical operations and approximations by fuzzy sets. Int. J. Gen Syst 12(3), 193-226 (1986)

[11] Dubois, D., Prade, H.: Possibility Theory: An approach to computerized processing of uncertainty. Plenum Press, New-York (1988)

[12] Dubois, D., Prade, H., Smets, Ph.: A Definition of Subjective Possibility. Int. J. Approximate Reasoning 48(2), 352-364 (2008)

[13] Dubois, D., Prade, H., Smets, Ph.: New semantics for quantitative possibility theory. In: Benferhat, S., Besnard, Ph. (eds.) Proceedings of the 6th European Conference on Symbolic and Quantitative Approaches to Reasoning with Uncertainty (ECSQARU'01), Toulouse, France, 2001. Lecture Notes in Computer Science, vol. 2143, pp 410-421. Springer-Verlag (2001)

[14] Elouedi, Z., Mellouli, K.: Pooling dependent expert opinions using the theory of evidence. In: Proceedings of the Seventh Int. Conf. on Information Processing and Management of Uncertainty in Knowledge-Based Systems (IPMU'98), Paris, France, 1998. Congrès Internationaux, vol. 1, pp 32-39. E.D.K., France (1998)

[15] Haenni, R.: Uncover Dempster's rule where it is hidden. In: Proceedings of the 9th International Conference on Information Fusion, Florence, Italy, July 2006.

[16] Hájek, P.: Deriving Dempster's rule. In: Valverde, L., Yager, R. R. (eds.) Uncertainty in Intelligent Systems, pp 75-83. North-Holland, Amsterdam (1993)

[17] Kallel, A., Le Hégarat-Mascle, S.: Combination of partially non-distinct beliefs: The cautious-adaptive rule. Int. J. Approximate Reasoning 50(7), 1000-1021 (2009) 
[18] Klawonn, F., Schwecke, E.: On the axiomatic justification of Dempster's rule of combination. Int. J. Intell. Syst. 7(5), 469-478 (1992)

[19] Klawonn, F., Smets, Ph.: The dynamic of belief in the transferable belief model and specialization-generalization matrices. In: Dubois, D., Wellman, M.P., D'Ambrosio, B., Smets, Ph. (eds.) Proceedings of the 8th conference on Uncertainty in Artificial Intelligence (UAI'92), pp 130-137. Morgan Kaufmann, San Meteo, CA (1992)

[20] Klement, E. P., Mesiar, R., Pap, E.: Triangular norms. Kluwer, Dordrecht (2000)

[21] Kohlas, J., Shenoy, P. P.: Computation in Valuation Algebras. In: Gabbay, D. M., Smets, Ph. (eds.) Handbook of Defeasible Reasoning and Uncertainty Management Systems: Algorithms for Uncertainty and Defeasible Reasoning, vol. 5, pp 5-39. Kluwer, Dordrecht (2000)

[22] Ling, X. N., Rudd, W. G.: Combining opinions from several experts. Applied Artificial Intelligence 3, 439-452 (1989)

[23] Liu, L. A theory of Gaussian belief functions. Int. J. of Approximate Reasoning 14(2-3), 95-126 (1996)

[24] Mercier, D., Cron, G., Denœux, T., Masson, M.-H.: Decision fusion for postal address recognition using belief functions. Expert Systems with Applications 36(3), 5643-5653 (2009)

[25] Pichon, F., Denœux, T.: A new justification of the unnormalized Dempster's rule of combination from the Least Commitment Principle. In: Proceedings of the 21th Florida Artificial Intelligence Research Society Int. Conference (FLAIRS'08), Special Track on Uncertain Reasoning, Coconut Grove, Florida, USA, pp 666-671, 15-17 May (2008)

[26] Pichon, F., Denœux, T.: T-norm and Uninorm-Based Combination of Belief Functions. In: Proceedings of the North American Fuzzy Information Processing Society Int. Conference (NAFIPS'08), New York, USA, 19-22 May (2008).

[27] Pichon, F., Denœux, T.: A new singular property of the unnormalized Dempster's rule among uninorm-based combination rules. In: Magdalena, L., Ojeda-Aciego, M., Verdegay, J.L. (eds) Proceedings of the 12th Int. Conf. on Information Processing and Management of Uncertainty in Knowledge-Based Systems (IPMU'08), pp 314321, Torremolinos (Malaga), Spain, 22-27 June (2008) 
[28] Quost, B., Denœux, T., Masson, M.-H.: Pairwise classifier combination using belief functions. Pattern Recognition Letters 28(5), 644-653 (2007)

[29] Quost, B., Denœux, T., Masson, M.-H.: Adapting a combination rule to nonindependent information sources. In: Magdalena, L., Ojeda-Aciego, M., Verdegay, J.L. (eds) Proceedings of the 12th Int. Conf. on Information Processing and Management of Uncertainty in Knowledge-Based Systems (IPMU'08), pp 448-455, Torremolinos (Malaga), Spain, 22-27 June (2008)

[30] Quost, B., Denœux, T., Masson, M.-H.: Refined classifier combination using belief functions. In: Proceedings of the 11th Int. Conf. on Information Fusion (FUSION'08), pp 776-782, Cologne, Germany, 30 June - 03 July (2008)

[31] Ristic, B., Smets, Ph.: Target identification using belief functions and implication rules. IEEE Transactions on Aerospace and Electronic Systems 41(3), 1097-1103 (2005)

[32] Sentz, K., Ferson, S.: Combination of evidence in Dempster-Shafer Theory. Technical report, SANDIA Tech. Report, SAND2002-0835 (2002)

[33] Shafer, G.: A mathematical theory of evidence. Princeton University Press, Princeton, N.J. (1976)

[34] Shenoy, P. P.: Conditional independence in valuation-based systems. Int. J. Approximate Reasoning 10, 203-234 (1994)

[35] Smets, Ph.: Un modèle mathématico-statistique simulant le processus du diagnostic médical. PhD thesis (in French), Université Libre de Bruxelles, Brussels, Belgium (1978)

[36] Smets, Ph.: The Combination of Evidence in the Transferable Belief Model. IEEE Transactions on Pattern Analysis and Machine Intelligence 12(5), 447-458, (1990)

[37] Smets, Ph.: Belief functions: the disjunctive rule of combination and the generalized Bayesian theorem. Int. J. Approximate Reasoning 9, 1-35 (1993)

[38] Smets, Ph.: What is Dempster-Shafer's model ? In: Yager, R. R., Kacprzyk, J., Fedrizzi, M. (eds.) Advances in the Dempster-Shafer theory of evidence, pp 5-34. Wiley, New-York (1994)

[39] Smets, Ph.: The canonical decomposition of a weighted belief. In: Proceedings of the 14th Int. Joint Conf. on Artificial Intelligence (IJCAI'95), San Mateo, California, USA, 1995, pp 1896-1901. Morgan Kaufmann (1995) 
[40] Smets, Ph.: The $\alpha$-junctions: combination operators applicable to belief functions. In: Gabbay, D.M., Kruse, R., Nonnengart, A., Ohlbach, H.J. (eds.), Proceedings of the First International Joint Conference on Qualitative and Quantitative Practical Reasoning (ECSQARU-FAPR'97), Bad Honnef, Germany, 1997. Lecture Notes in Computer Science, vol. 1244, pp 131-153. Springer (1997)

[41] Smets, Ph.: Analyzing the combination of conflicting belief functions. Information Fusion 8(4), 387-412 (2007)

[42] Smets, Ph.: The Transferable Belief Model for quantified belief representation. In: Gabbay, D. M., Smets, Ph. (eds.), Handbook of Defeasible Reasoning and Uncertainty Management Systems, vol. 1, pp 267-301. Kluwer, Dordrecht (1998)

[43] Smets, Ph., Kennes, R.: The Transferable Belief Model. Artificial Intelligence 66, 191-243 (1994)

[44] Smets, Ph., Kruse, R.: The Transferable Belief Model for Belief Representation. In: Motro, A., Smets, Ph. (eds.) Uncertainty Management in Information Systems: From Needs to Solution, pp 343-368. Kluwer Academic Publishers, Boston (1996)

[45] Yager, R. R.: An Introduction to Applications of Possibility Theory. Human Systems Management 3, 246-269 (1983)

[46] Yager, R. R.: The entailment principle for Dempster-Shafer granules. Int. J. Intell. Syst. 1, 247-262 (1986)

[47] Yager, R. R., Rybalov, A.: Uninorm aggregation operators. Fuzzy Sets and Systems 80, 111-120 (1996) 